\title{
Can we rely on out-of-hospital blood samples? A prospective interventional study on the pre-analytical stability of blood samples under prehospital emergency medicine conditions
}

\author{
Johannes Prottengeier ${ }^{1 *}$ (D) Nicola Jess ${ }^{2}$, Frank Harig ${ }^{3}$, Christine Gall ${ }^{4}$, Joachim Schmidt ${ }^{1}$ and Torsten Birkholz ${ }^{1}$
}

\begin{abstract}
Background: Prehospital intravenous access provides the opportunity to sample blood from an emergency patient at the earliest possible moment in the course of acute illness and in a state prior to therapeutic interventions. Our study investigates the pre-analytical stability of biomarkers in prehospital emergency medicine and will answer the question whether an approach of blood sampling out in the field will deliver valid laboratory results.
\end{abstract}

Methods: We prepared pairs of blood samples from healthy volunteers and volunteering patients post cardio-thoracic surgery. While one sample set was analysed immediately, the other one was subjected to a worse-than-reality treatment of $60 \mathrm{~min}$ time-lapse and standardized mechanical forces outside of the hospital through actual ambulance transport. We investigated 21 parameters comprising blood cells, coagulation tests, electrolytes, markers of haemolysis and markers of cardiac ischemia. Bland-Altman analysis was used to investigate differences between test groups. Differences between test groups were set against the official margins of test accuracy as given by the German Requirements for Quality Assurance of Medical Laboratory Examinations.

Results: Agreement between immediate analysis and our prehospital treatment is high as demonstrated by Bland-Altman plotting. Mechanical stress and time delay do not produce a systematic bias but only random inaccuracy. The limits of agreement for the tested parameters are generally within clinically acceptable ranges of variation and within the official margins as set by the German Requirements for Quality Assurance of Medical Laboratory Examinations.

Discussion: We subjected blood samples to a standardized treatment marking a worse-than-reality scenario of prehospital time delay and transport. Biomarkers including indicators of myocardial ischemia showed high pre-analytical stability.

Conclusion: We conclude the validity of blood samples from a prehospital environment.

Keywords: Blood sampling, Sample handling, Preanalytical stability, Prehospital emergency medicine

\footnotetext{
* Correspondence: johannes.prottengeier@kfa.imed.uni-erlangen.de

'Department of Anaesthesiology, Erlangen University Hospital,

Krankenhausstrasse 12, 91054 Erlangen, Germany

Full list of author information is available at the end of the article
} 


\section{Background}

Laboratory tests from blood samples are a major component in the diagnostic work-up of emergency patients $[1,2]$. Usually the required blood samples are drawn inside the emergency department (ED) and there is a general consensus, that samples should be analysed as soon as possible and handled carefully [3-5]. Whenever a patient is being cared for by emergency medical services (EMS) prior to his admission to the ED, there arises an opportunity and unsolved question at the same time: EMS will frequently establish venous access $[6,7]$. This could very well be utilized to secure blood specimens back-to-back to a patient's first contact with medical help. Firstly, such samples would produce results representative of the patient's condition before any intervention: before infusion of fluids and haemodilution, before heparin and anticoagulation, before diuretics and changes to electrolytes - to mention a few iatrogenic alterations. Secondly they would provide additional insight into the course of dynamic biomarkers in scenarios such as myocardial infarction [8]. And finally, prehospital sampling may save time in the diagnostic process as time consumption for blood sampling adjacent to the process of venous cannulation may be smaller than a second procedure performed inside the ED, especially considering the inversion of the patient-staff ratio from the prehospital to the intrahospital setting.

But are those presumed advantages real? Up to date no data exists to evaluate the clinical value of prehospital blood sampling. Even less, literature provides no answer to the more basic question, whether those laboratory values relevant to emergency medicine show the necessary preanalytical stability to allow for reliable testing-if they were gathered in a prehospital setting.

The aim of our study was to subject blood samples to a standardized "worst-case-scenario" prehospital treatment of mechanical forces and time delay before laboratory testing. We investigated the preanalytical stability of biomarkers frequently used in emergency diagnostics compared to an immediate analysis. This included myocardial enzymes, complete blood counts and tests of haemostasis, haemolysis, electrolytes and different proteins. Owing to its key role in the work-up of "chestpain" we focused on the changes of troponin levels as our primary parameter of interest.

\section{Methods \\ Blood donors}

This study was approved by the ethics board of the University of Erlangen-Nuremberg. Blood sampling took place at the Erlangen University Hospital. Samples where provided by healthy volunteers (physicians, nurses, paramedics) and volunteering scheduled patients post major cardio-thoracic surgery. All volunteers gave their informed consent well before the investigations. The composition of our study population guaranteed that biomarkers covered a wide range of values from physiological (in healthy volunteers) to highly pathological (e.g. elevated cardiac enzymes in patients post cardiotomy).

\section{Blood sampling}

All samples were collected under standardized, stringently timed conditions and adherence to the syringe manufacturers' product manuals and the hospital's standard operating procedures of hygiene and safety. Only venous blood was obtained. Specimens for blood gas analysis were contained in "safePico Aspirators" (Radiometer Medical ApS, Brønshøj, Denmark) and all other specimen in "S-Monovettes" (Sarstedt AG, Nümbrecht, Germany). Table 1 shows the allocation of parameters to their specific syringe. Analysis took place in the University Hospital's laboratories with the exception of blood gas analyses, which were performed on the Department of Anaesthesia's own ABL 800Flex analysers (Radiometer Medical ApS, Brønshøj, Denmark). Table 2 provides an overview of analysers, analytical methods and legally required analytical accuracy for each parameter.

Table 1 List of parameters and sample syringes

\begin{tabular}{|c|c|c|c|c|c|}
\hline Type of syringe & $\begin{array}{l}\text { S-Monovette/red } \\
\text { Haematology }\end{array}$ & $\begin{array}{l}\text { S-Monovette/green } \\
\text { Coagulation profile }\end{array}$ & $\begin{array}{l}\text { S-Monovette/white } \\
\text { Clotting Activator/Serum }\end{array}$ & $\begin{array}{l}\text { S-Monovette/orange } \\
\text { Lithium Heparin/Serum }\end{array}$ & $\begin{array}{l}\text { safePICO Aspirator } \\
\text { Blood-Gas-Analysis }\end{array}$ \\
\hline Additive & Potassium-EDTA & Citrate & Clotting Activator & Lithium Heparin & 80 IU Heparin \\
\hline \multirow{6}{*}{$\begin{array}{l}\text { Parameters (alphabetic order) } \\
\text { of laboratory testing }\end{array}$} & Haemoglobin & Antithrombin III & Alpha-HBDH & Alpha-HBDH & Calcium/Ca++ \\
\hline & Leukocytes & $\begin{array}{l}\text { Activated Partial } \\
\text { Thromboplastin Time (aPTT) }\end{array}$ & Creatinine & Creatinine & Chloride/Cl- \\
\hline & Thrombocytes & Fibrinogen & Creatin Kinase/CK & Creatin Kinase/CK & Potassium/K $\mathrm{K}^{+}$ \\
\hline & & $\begin{array}{l}\text { International Normalized } \\
\text { Ratio (INR) }\end{array}$ & Free Haemoglobin & Free Haemoglobin & Sodium/Na ${ }^{+}$ \\
\hline & & & $\begin{array}{l}\text { Glutamic Oxaloacetic } \\
\text { Transaminase }\end{array}$ & $\begin{array}{l}\text { Glutamic Oxaloacetic } \\
\text { Transaminase }\end{array}$ & \\
\hline & & & & Troponin I & \\
\hline
\end{tabular}




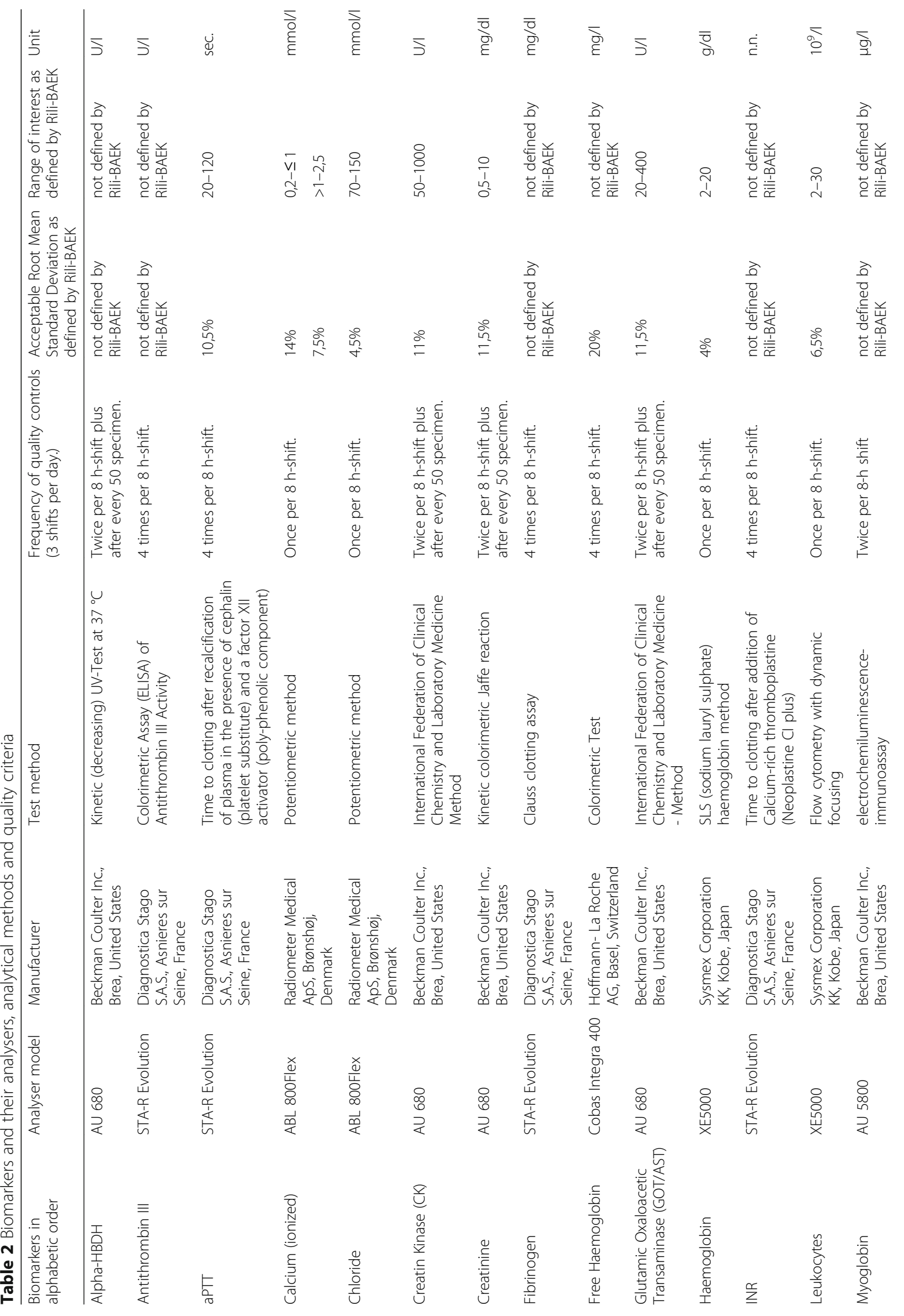




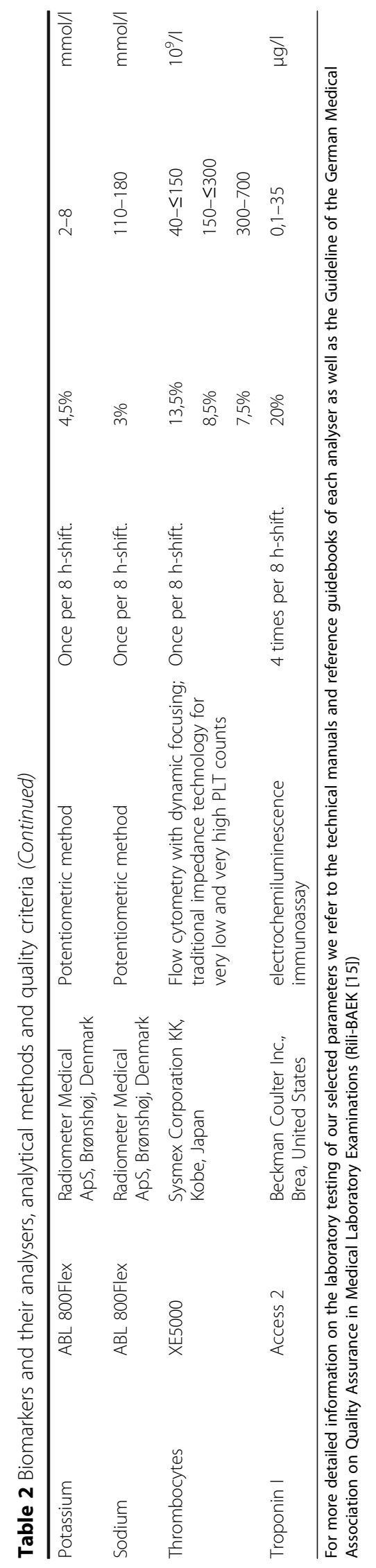


We generated two equal sets of syringes from every volunteer. Parameters can be divided in two groups: Group 1 represents a shortlist of biomarkers highly relevant to emergency medicine. Group 2 (items in italics in Table 1) represent markers of haemolysis which may falsify other test procedures.

Each set of samples was allotted to one of two different treatment conditions.

\section{Control Group (CG)}

All specimen were immediately transported to analysis by foot within $10 \mathrm{~min}$ of sampling. All specimens were subject to room temperature as provided by the hospital's climate control system.

\section{Prehospital Group (PHG)}

Samples were analysed after a time lapse of 60 min during which they underwent a standardized scenario of mechanical stress (see below). During transport all samples were kept lying flat in a cooler box (Coleman Inc., Wichita, United States) tempered to the intensive care unit's $23{ }^{\circ} \mathrm{C}$ standard room temperature, which is maintained by a climate control system. Temperature of the test compartment was monitored end-to-end and samples were not subject to external heating or cooling. By these means of storage, samples were also shielded from solar radiation.

\section{Prehospital scenario}

Samples allotted to the PHG were transferred to an ambulance truck (1998 Sprinter, Model 312 D, Mercedes Benz, Stuttgart, Germany. It was the oldest and most worn down model available from local emergency services at that time.). The Cooler Box was placed on the worktop inside the patient's cabin. Samples were sent onto a $15 \mathrm{~km}$ and $30 \mathrm{~min}$ long drive in and around the city of Erlangen, which consisted of city traffic, dirt tracks and larger state roads. Driving mode (gear), velocity, and thus time consumption and the quantity of mechanical forces were standardized through a dedicated driving protocol. The reproducibility of mechanical forces was evaluated in pretest trials using a Triaxial Vibration Meter VM30-H (Metra Mess- und Frequenztechnik, Radebeul, Germany) placed in the site of the syringes.

Under the infrastructural and geographic circumstances of German EMS, pre-hospital time requirements shall not exceed $60 \mathrm{~min}$. This time frame has originated from the "golden hour of shock" and has been adopted for timesensitive diagnoses such as multiple trauma, traumatic brain injury, stroke, sepsis, chest-pain and resuscitation. It is the upper limit for the pre-hospital phase of emergency care in Germany and is one of the main determinants for the allocation of EMS resources [9].

In context of these circumstances, our prehospital setup represented a worst-case scenario by far longer in duration and far more mechanically straining than average missions found in land-based EMS in Germany [10-13].

\section{Data analysis and statistics}

To analyse the effect of mechanical stress and time on blood samples, we use Bland-Altman plots to compare the measurements with the reference blood samples which were analysed immediately [14].

The solid line in the graph shows the mean of the differences. The dashed upper (lower) line shows the upper (lower) limit of agreement equal to mean $\pm 2 \mathrm{SD}$. Within these limits, typically lie $95 \%$ of the samples differences. The dotted lines represent the respective 95\% confidence intervals. As external margins for negligible differences, we use the relative differences specified for certain ranges of validity given by the Guidelines of the German Medical Association on quality assurance in medical laboratory testing (Rili-BAEK) [15]. The Rili-BAEK is the official benchmarking guideline of German medical laboratories. The validity of test results is strictly limited by predefined margins of error. Therefore we postulate that results must be considered as equal, should their differences lie within the set margins of the Rili-BAEK.

Comparing Bland-Altman plots for absolute and relative differences confirmed our choice of absolute differences as then the points better fit the horizontal limits of agreement along the range of the $x$-axis. Thus, the tolerated difference given by Rili-BAEK [15] is not constant over $\mathrm{x}$ but shown by sloping lines.

Analyses were performed in $\mathrm{R}$ 3.2.4 [ $\mathrm{R}$ Core Team (2013). R: A language and environment for statistical computing. R Foundation for Statistical Computing, Vienna, Austria. URL http://www.R-project.org/].

\section{Results}

We prepared 134 sample sets and selected 21 biomarkers for Bland-Altman analysis (Table 3). Factors such as analyser malfunction, treatment against protocol (dropping of syringes, inadequate filling heights etc.) led to the exclusion of some samples. The number of complete data sets for each parameter is documented.

The effect of our prehospital scenario compared to immediate testing is demonstrated by the Bland-Altman plots of each parameter. We present in this manuscript a selection of plots in Figs. 1, 2, 3, 4, 5 and 6. Plots for 8 other analysed parameters can be found as Additional files 1, 2, 3, 4, 5, 6, 7 and 8 (Figures S7-S14). Table 3 provides a comprehensive overview of each parameter, means of differences, standard deviations and confidence intervals between Groups CG and PHG. The estimated means of differences are always negligible with a small $95 \%$-CI, i.e. mechanical stress does not produce a systematic bias but only random inaccuracy. The limits of agreement are universally within clinically acceptable 


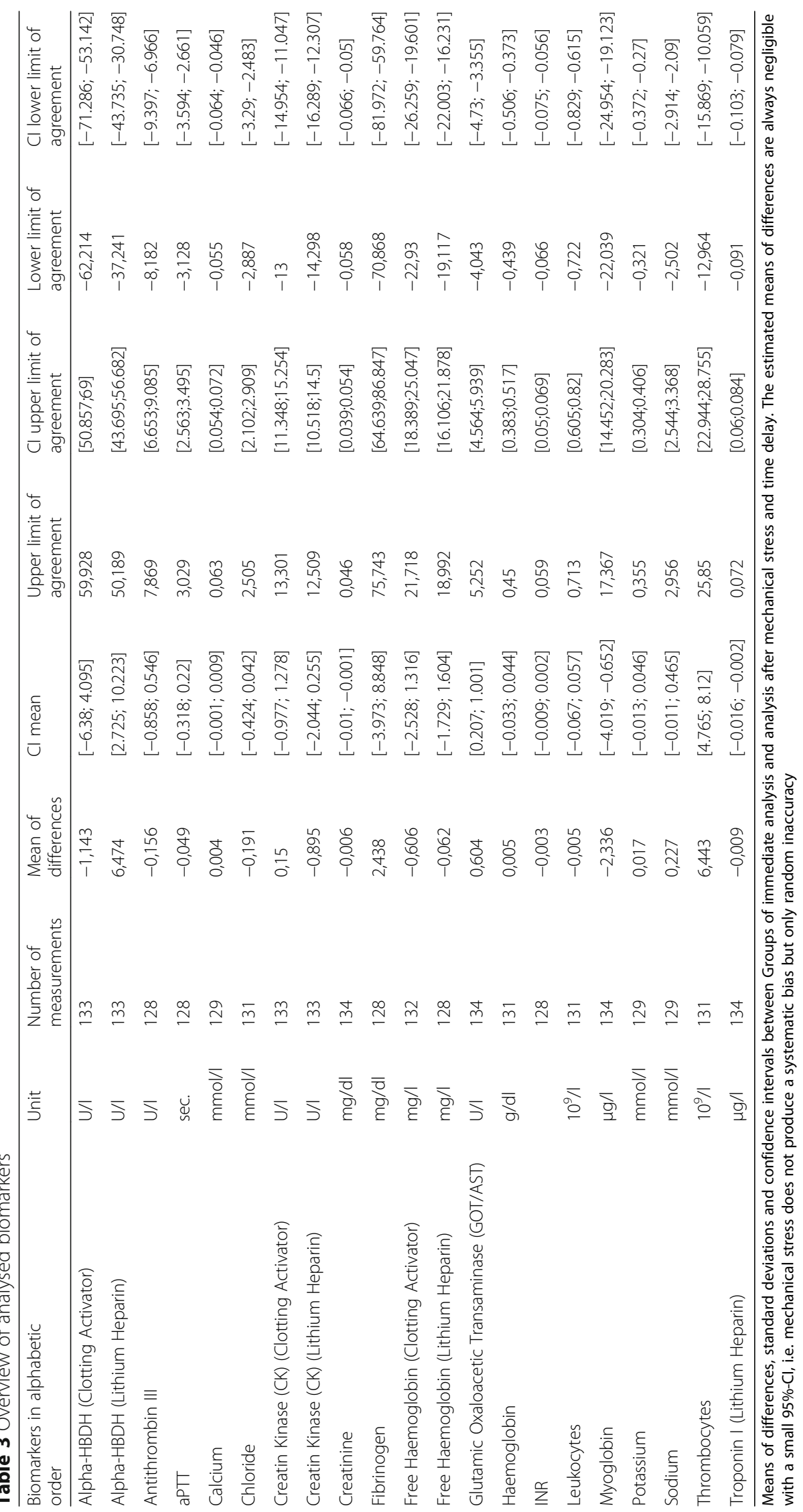



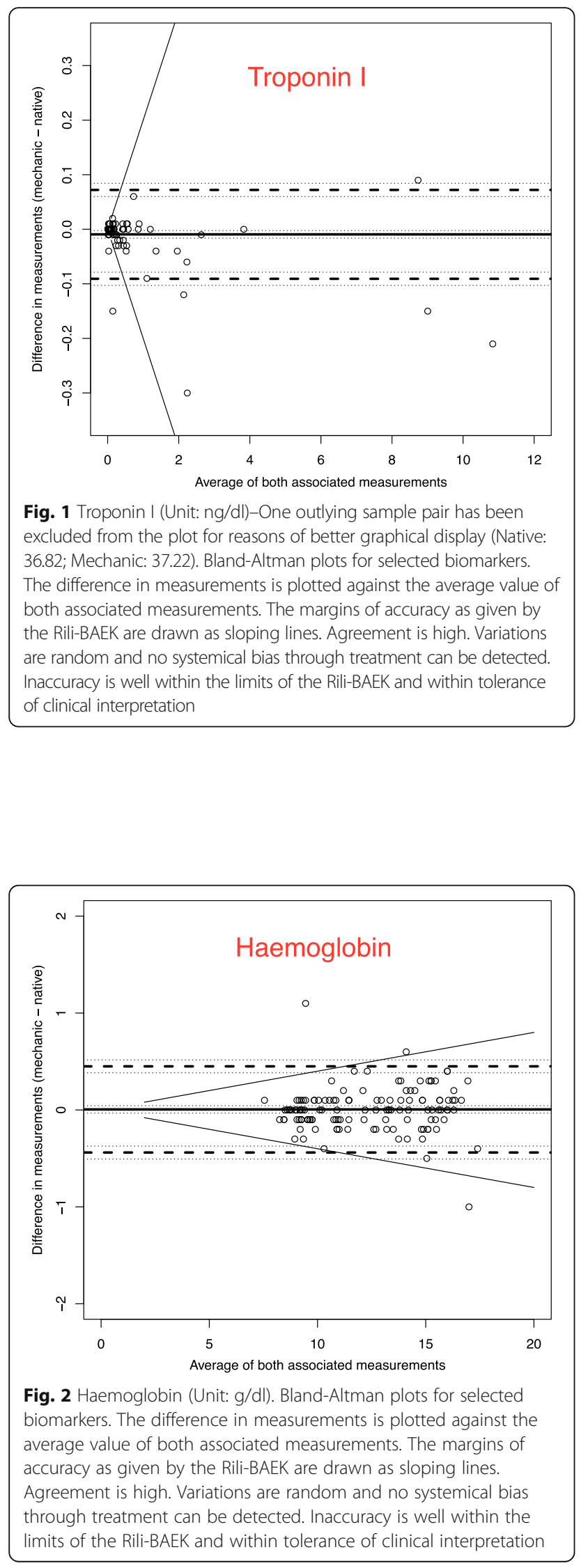

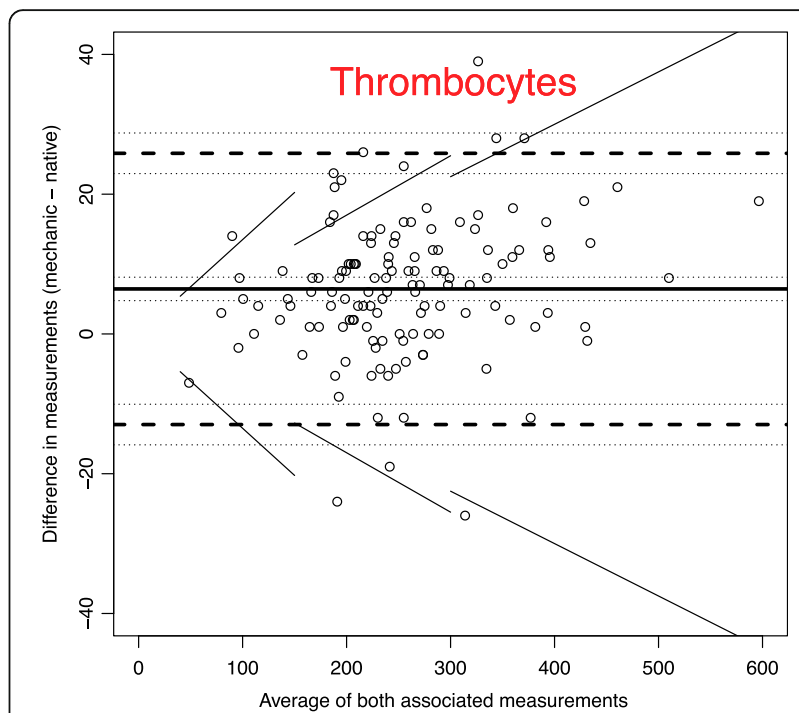

Fig. 3 Thrombocytes (Unit: 10\% /l). Bland-Altman plots for selected biomarkers. The difference in measurements is plotted against the average value of both associated measurements. The margins of accuracy as given by the Rili-BAEK are drawn as sloping lines. Agreement is high. Variations are random and no systemical bias through treatment can be detected. Inaccuracy is well within the limits of the Rili-BAEK and within tolerance of clinical interpretation

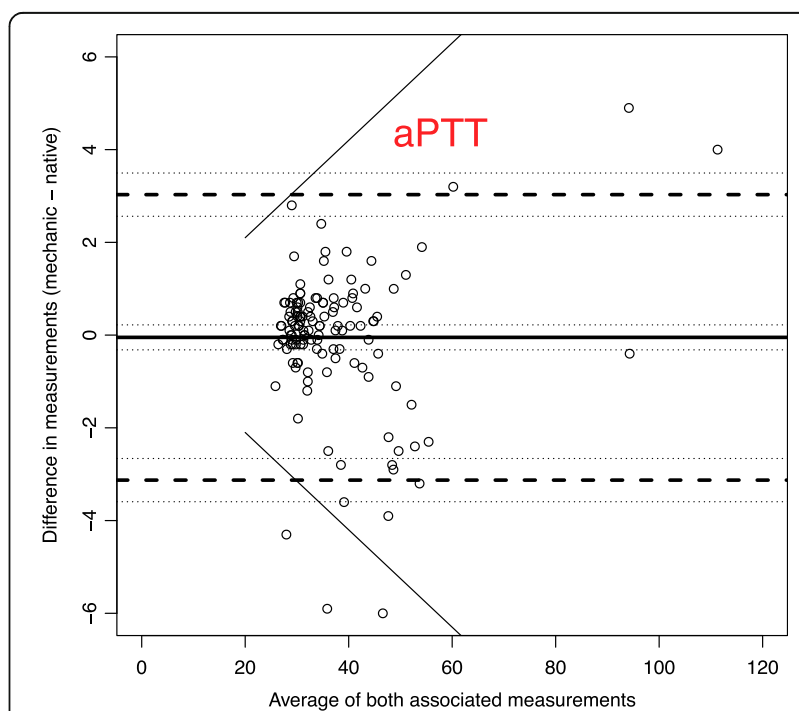

Fig. 4 Activated Partial Thromboplastin Time - aPTT (Unit: sec.). Bland-Altman plots for selected biomarkers. The difference in measurements is plotted against the average value of both associated measurements. The margins of accuracy as given by the Rili-BAEK are drawn as sloping lines. Agreement is high. Variations are random and no systemical bias through treatment can be detected. Inaccuracy is well within the limits of the Rili-BAEK and within tolerance of clinical interpretation 


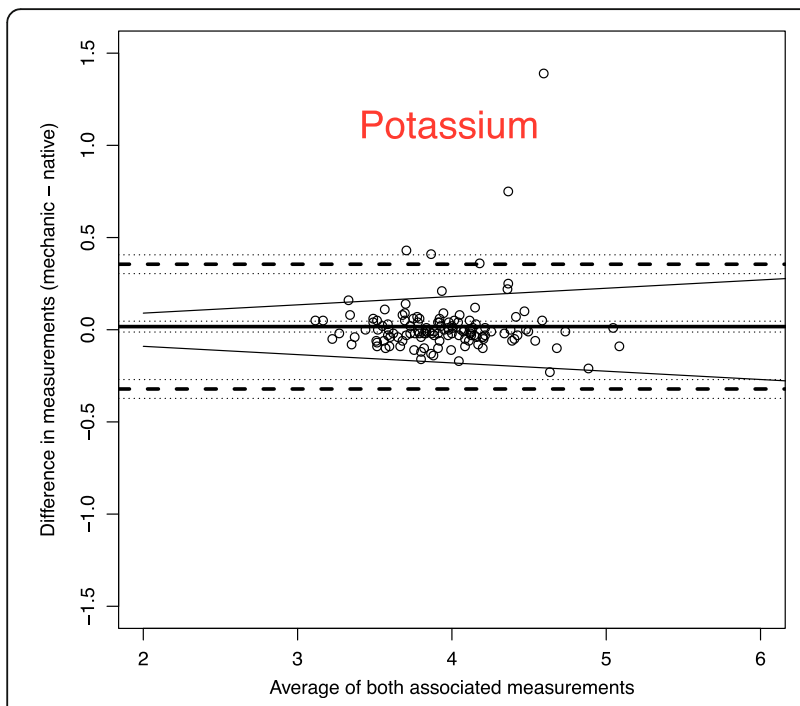

Fig. 5 Potassium (Unit: mmol/l). Bland-Altman plots for selected biomarkers. The difference in measurements is plotted against the average value of both associated measurements. The margins of accuracy as given by the Rili-BAEK are drawn as sloping lines. Agreement is high. Variations are random and no systemical bias through treatment can be detected. Inaccuracy is well within the limits of the Rili-BAEK and within tolerance of clinical interpretation ranges of variation. Alterations are mostly within the official margin of accuracy as set by the Rili-BAEK. Troponin I showed comparable results when compared between immediate analysis and analysis after the combination of time delay and mechanical stress. Among the 134 samples, $47 / 134=35 \%$ of the troponin values lie within the interval from 0.1 to $35 \mathrm{ng} / \mathrm{dl}$ which is the range addressed by the Rili-BAEK. Only for two samples, the relative difference was more than $0.2 \mathrm{ng} / \mathrm{dl}$, precisely 0.3 and $0.21 \mathrm{ng} / \mathrm{dl}$. For $15(=15 / 47=32 \%)$ of these 47 values, there was no measurable difference at all, i.e. the measurement after prehospital treatment was exactly like the immediately analysed one. For the rest of the values, the relative difference was less or equal to $0.15 \mathrm{ng} / \mathrm{dl}$. Thus, for $45 / 47$ $=95,7 \%$ (CI $[89.97 \% ; 100 \%])$ of the samples, the relative difference is easily acceptable. For $87 / 134=65 \%$ of the samples, the troponin measurements are lower than $0.1 \mathrm{ng} / \mathrm{dl}$. For $78(=89.655 \%, \mathrm{CI}=[83.256 \% ; 96.1 \%]) \mathrm{sam}-$ ples, the deviation is Zero or less than the technical capabilities of measurement, respectively. For seven samples, the absolute differences are equal to $0.01 \mathrm{ng} / \mathrm{dl}$. For the remaining two samples, they are 0.04 and $0.4 \mathrm{ng} / \mathrm{dl}$.

Haemolysis as a potent interference factor with many chromage laboratory tests was investigated and excluded in relevant levels by investigations into alpha-HBDH and free haemoglobin in serum (Fig. 6 and Additional files 6, 7 and 8: Figures S12-S14).

\section{Discussion}

Emergency services regularly represent the first line of contact between medical care and patients. In many cases, circumstances would allow for the sampling of blood in the early stages of acute illness. While it is conceivable that such an approach might have clinical benefits, there is little data to proof the pre-analytical validity of biomarkers achieved this way. In our study we simulated the prehospital fate of blood samples and investigated changes to test results when compared to immediate analysis. We improvised a combination of time delay and mechanical stress that resulted in a sample exposition worse than what would be expected in German EMS on a regular basis.

We found that our exposition of specimens towards time delay and mechanical stress caused only small changes to the parameters, as visualised in the Bland-Altman-Plots. However, the seemingly simple question whether the plotted differences may be considered as "same or different" cannot be answered by a universally accepted formula. The interpretation of bare values actually depends on the overall clinical context and many factors more.

The definition of "analytical validity" of the laboratory test itself also deals with this challenge and provides guidance for our dilemma. Definitions and margins 
derive from technical aspects. They are generally accepted and uncoupled from subjective interpretation. Even more so, they are given at a level of precision that guarantees a reliable foundation for the diagnostic process in general. Stringent quality control is mandatory and defines clear margins for the accuracy of analytical testing for each biomarker (Rili-BAEK) [15]. As a consequence it has to be postulated, that test results with differences between them that lie within these limits of test accuracy must be accepted as equal technically and clinically.

Within our data set the differences between treatment groups generally lie within those margins of test accuracy. Therefore results after prehospital treatment can be considered as clinically equal to those from immediate analysis. The pre-analytical validity is given under conditions of a worst-case scenario and therefore we postulate even smaller inaccuracies under average mission circumstances in Germany. Stability is given in all types of syringes. It is demonstrated for cell counts, coagulation profiles, electrolyte levels and protein serum biomarkers. Our assortment of tests represents some of the most relevant biomarkers in emergency medicine and covers a wide range of disorders.

Haemolysis may interfere with chromagen tests, but has been ruled out as a confounder by stable results for alpha-HBDH and free haemoglobin during our experiments $[16,17]$.

These findings are in accordance with previous studies of pre-analytical stability of biomarkers in other surroundings. Troponin shows preanalytical validity in a variety of maltreatment scenarios such as prolonged or sub-par storage and repeated freeze-thaw cycles [18-20]. However, troponin is a protein of limited metabolism within the confinements of a blood sample and results may vary with biomarkers of different biological properties.

Rotational thromboelastometry for example represents a multifactorial functional test that is highly susceptible to mechanical interference during analysis, and has been shown to be subject to pre-analytical influences as well. Transport of samples through pneumatic tube systems led to statistically significant alterations of test results. However, those changes were all within limits of what the investigators defined as clinical irrelevance [21, 22].

Furthermore, some biomarkers need to be excluded from analysis owing to biophysical considerations. Volatile solubles such as oxygen and carbon dioxide will rapidly alter their concentrations with clinical relevance only minutes after sampling [23, 24].

In the end the clinical relevance of our study derives from three points. 1. Our analysis brings alterations into the accepted context of analytical accuracy as outlined by the quality-assurance guidelines of the "Rili-BAEK". 2. Treatment of samples represents a worst-case scenario and suggests less error with normal treatment. 3. Our choice of parameters reflects on relevance to emergency medicine and excludes those a priori unsuitable due to biophysical reasons.

Naturally there are some limitations to our study. As a first, our specific scenario to test sample integrity represents only one combination of stressors out of many thinkable. Our main focus lay on time delay and mechanical forces as possible factors of interference. However, other external circumstances may influence preanalytical stability as well such as subpar conditions for blood sampling itself, extreme variations in temperature and exposure to solar radiation. As pre-hospital emergency medicine regularly takes place in an improvised outdoor environment, these influences should be considered and investigated in future studies. However, our study also demonstrates, that low-tech solutions such as the use of a cooler-box for sample storage can substantially limit the impact of environmental elements.

Secondly, we have to admit that specimen treatment might differ in nuances from ride to ride - in spite of all steps taken to provide for reproducibility. However, these limitations seem agreeable when compared to the alternative of-highly standardizable, but totally artificial-mechanical stress generated by vibrating devices inside a laboratory.

Thirdly, our exposition scenario is aligned towards transportation times found in the well-developed EMSinfrastructure of a densely-populated country. It does not cover very long sample transportation times beyond $60 \mathrm{~min}$ as they might be found in geographically challenging settings or under conditions of limited health-care infrastructure [25].

As a forth, we can only draw conclusions from parameters within the range of our test samples. While it is biologically unlikely that extremely high or low concentrations of biomarkers will have different reactions to our scenario, the absence of extreme values in our data set does not allow anything but speculation for such outliers.

Finally, our investigation focused on a selection of parameters and methods of analysis and we can only provide data for those within this collection.

\section{Conclusion}

Prehospital emergency medicine offers the possibility to sample blood in the early stages of acute illness. Our study demonstrates the pre-analytical stability and validity of samples of such origin even after time delay and transport outside of the hospital. The data set includes markers of myocardial ischemia such as troponin and creatine kinase, next to blood cells, electrolytes and coagulation profiles. Keeping in mind the limitations of this investigation, it seems worthwhile to investigate through future studies, 
the possible benefits to patient outcome deriving from out-of-hospital blood sampling.

\section{Additional files}

Additional file 1: Figure S1. Leukocytes (Unit: $\left.10^{9} / \mathrm{I}\right)$. Bland-Altman plots for selected biomarkers. The difference in measurements is plotted against the average value of both associated measurements. The margins of accuracy as given by the Rili-BAEK are drawn as sloping lines. Agreement is high. Variations are random and no systemical bias through treatment can be detected. Inaccuracy is well within the limits of the Rili-BAEK and within tolerance of clinical interpretation. (PDF $37 \mathrm{~kb}$ )

Additional file 2: Figure S2. INR (Unit: not applicable). Bland-Altman plots for selected biomarkers. The difference in measurements is plotted against the average value of both associated measurements. The margins of accuracy as given by the Rili-BAEK are drawn as sloping lines. Agreement is high. Variations are random and no systemical bias through treatment can be detected. Inaccuracy is well within the limits of the Rili-BAEK and within tolerance of clinical interpretation. (PDF $36 \mathrm{~kb}$ )

Additional file 3: Figure S3. Creatin Kinase in serum with clotting activator (Unit: U/I). Bland-Altman plots for selected biomarkers. The difference in measurements is plotted against the average value of both associated measurements. The margins of accuracy as given by the Rili-BAEK are drawn as sloping lines. Agreement is high. Variations are random and no systemical bias through treatment can be detected. Inaccuracy is well within the limits of the Rili-BAEK and within tolerance of clinical interpretation. (PDF $63 \mathrm{~kb}$ )

Additional file 4: Figure S4. Creatin Kinase in Lithium-Heparin preserved serum (Unit: U/I). Bland-Altman plots for selected biomarkers. The difference in measurements is plotted against the average value of both associated measurements. The margins of accuracy as given by the Rili-BAEK are drawn as sloping lines. Agreement is high. Variations are random and no systemical bias through treatment can be detected. Inaccuracy is well within the limits of the Rili-BAEK and within tolerance of clinical interpretation. (PDF 94 kb)

Additional file 5: Figure S5. Creatinine (Unit: $\mathrm{mg} / \mathrm{dl}$ ). Bland-Altman plots for selected biomarkers. The difference in measurements is plotted against the average value of both associated measurements. The margins of accuracy as given by the Rili-BAEK are drawn as sloping lines. Agreement is high. Variations are random and no systemical bias through treatment can be detected. Inaccuracy is well within the limits of the Rili-BAEK and within tolerance of clinical interpretation. (PDF $154 \mathrm{~kb}$ )

Additional file 6: Figure S6. Free Haemoglobin in serum with clotting activator (Unit: $\mathrm{mg} / \mathrm{l})$. Bland-Altman plots for selected biomarkers. The difference in measurements is plotted against the average value of both associated measurements. The margins of accuracy as given by the Rili-BAEK are drawn as sloping lines. Agreement is high. Variations are random and no systemical bias through treatment can be detected. Inaccuracy is well within the limits of the Rili-BAEK and within tolerance of clinical interpretation. (PDF $286 \mathrm{~kb}$ )

Additional file 7: Figure S7. Alpha-HBDH in serum with clotting activator (Unit: U/I) - Two outlying sample pairs have been excluded from the plot for reasons of better graphical display (No. 1: Native: 1532; Mechanic: 1529; No. 2: Native 357: Mechanic: 135). Bland-Altman plots for selected biomarkers. The difference in measurements is plotted against the average value of both associated measurements. The margins of accuracy as given by the Rili-BAEK are drawn as sloping lines. Agreement is high. Variations are random and no systemical bias through treatment can be detected. Inaccuracy is well within the limits of the Rili-BAEK and within tolerance of clinical interpretation. (PDF $566 \mathrm{~kb}$ )

Additional file 8: Figure S8. Alpha-HBDH in Lithium-Heparin preserved serum (Unit: U/I)-One outlying sample pair has been excluded from the plot for reasons of better graphical display (Native: 1526; Mechanic: 1534). Bland-Altman plots for selected biomarkers. The difference in measurements is plotted against the average value of both associated measurements. The margins of accuracy as given by the Rili-BAEK are drawn as sloping lines. Agreement is high. Variations are random and no systemical bias through treatment can be detected. Inaccuracy is well within the limits of the Rili-BAEK and within tolerance of clinical interpretation. (PDF $38 \mathrm{~kb}$ )

\section{Abbreviations}

CG: Control Group; ED: Emergency department; EMS: Emergency medical service; PHG: Pre-Hospital-Group; Rili-BAEK: Guidelines of the German Medical Association on quality assurance in medical laboratory testing

\section{Acknowledgements}

We thank Gabi Göhring-Waldeck and Daniel Wohlfahrt for their excellent technical assistance. We thank Hans Parsch, Erwin Strasser and all their laboratory technicians for their excellent work in parameter analysis. We thank Jens Kasberger for providing us the Ambulance Truck for our test drives.

Finally, we thank all volunteers for their contribution to our study.

\section{Funding}

This study was funded by a science grant from the Arbeiter-Samariter-Bund Bayern Notfallhilfe.

\section{Availability of data and materials}

The datasets generated and analyzed during the current study are available from the corresponding author on reasonable request.

\section{Authors' contributions}

JP conceived of and conducted the study, performed the statistical analysis and is the main author of the manuscript. TB participated in the study's design and helped to draft the manuscript. $\mathrm{NJ}$ and FH participated in the coordination of the study and revised the manuscript critically for its intellectual content. CG performed the statistical analysis and revised the manuscript critically for its intellectual content. All authors read and approved the final manuscript.

\section{Competing interests}

All of the authors declare that they have no competing interests.

\section{Consent for publication}

Detailed, written, informed consent was obtained from all blood sample donors prior to all experiments.

\section{Ethics approval and consent to participate}

The Ethics Committee of Friedrich-Alexander-University, Erlangen, Germany (Chairperson Prof P. Betz) approved of this study under reference Number 319_14B.

\section{Author details}

'Department of Anaesthesiology, Erlangen University Hospital, Krankenhausstrasse 12, 91054 Erlangen, Germany. ${ }^{2}$ Department of Child and Adolescent Psychiatry, Psychosomatics, and Psychotherapy, Ludwig-Maximilians-University, Munich, Germany. ${ }^{3}$ Department of Cardiac Surgery, Erlangen University Hospital, Erlangen, Germany. ${ }^{4}$ Department of Medical Informatics, Biometry and Epidemiology, Friedrich Alexander University, Erlangen-Nuremberg, Erlangen, Germany.

Received: 18 August 2016 Accepted: 27 February 2017 Published online: 04 March 2017

\section{References}

1. Nguyen HB, Jaehne AK, Jayaprakash N, Semler MW, Hegab S, Yataco AC, Tatem G, Salem D, Moore S, Boka K, et al. Early goal-directed therapy in severe sepsis and septic shock: insights and comparisons to ProCESS, ProMISe, and ARISE. Crit Care. 2016;20(1):160.

2. Vaubourdolle M, Alvarez JC, Barbe F, Beaudeux JL, Boissier E, Caillon H, Chatron P, Joly-Guillou ML, Mailloux A. SFBC guidelines on critical care testing. Ann Biol Clin. 2016;74(2):130-55.

3. Davis MD, Walsh BK, Sittig SE, Restrepo RD. AARC clinical practice guideline: blood gas analysis and hemoximetry: 2013. Respir Care. 2013;58(10):1694-703.

4. Burnett RW, Covington AK, Fogh-Andersen N, Kulpmann WR, Maas AH, Muller-Plathe O, Siggaard-Andersen O, Van Kessel AL, Wimberley PD, Zijlstra WG. International Federation of Clinical Chemistry (IFCC). Scientific Division. 
Committee on pH, Blood Gases and Electrolytes. Approved IFCC recommendations on whole blood sampling, transport and storage for simultaneous determination of $\mathrm{pH}$, blood gases and electrolytes. Eur J Clin Chem Clin Biochem. 1995:33(4):247-53.

5. Lippi G, Banfi G, Church S, Cornes M, De Carli G, Grankvist K, Kristensen GB, Ibarz M, Panteghini M, Plebani M, et al. Preanalytical quality improvement. In pursuit of harmony, on behalf of European Federation for Clinical Chemistry and Laboratory Medicine (EFLM) Working group for Preanalytical Phase (WG-PRE). Clin Chem Lab Med. 2015;53(3):357-70.

6. van Schuppen H, Bierens J. Understanding the prehospital physician controversy. Step 1: comparing competencies of ambulance nurses and prehospital physicians. Eur J Emerg Med. 2011;18(6):322-7.

7. Cotton BA, Jerome R, Collier BR, Khetarpal S, Holevar M, Tucker B, Kurek S, Mowery NT, Shah K, Bromberg W, et al. Guidelines for prehospital fluid resuscitation in the injured patient. J Trauma. 2009;67(2):389-402.

8. Layfield C, Rose J, Alford A, Snyder SR, Apple FS, Chowdhury FM, Kontos MC, Newby LK, Storrow AB, Tanasijevic M, et al. Effectiveness of practices for improving the diagnostic accuracy of Non ST Elevation Myocardial Infarction in the Emergency Department: A Laboratory Medicine Best Practices systematic review. Clin Biochem. 2015;48(4-5):204-12.

9. Fischer M, Kehrberger E, Marung H, Moecke H, Prückner S, Trentzsch H, Urban B. The consensus document on emergency care for the general public during the pre-hospital phase and at the hospital. Notfall + Rettungsmedizin. 2016;19(5):387-95.

10. Schuster M, Pints M, Fiege M. Duration of mission time in prehospital emergency medicine: effects of emergency severity and physicians level of education. Emerg Med J. 2010;27(5):398-403.

11. Institut für Notfallmedizin und Medizinmanagement (INM). Trend- und Strukturanalyse des Rettungsdienstes in Bayern (TRUST). Munich: Klinikum der Universität München; 2015.

12. Garner AA, Mann KP, Poynter E, Weatherall A, Dashey S, Puntis M, Gebski V. Prehospital response model and time to CT scan in blunt trauma patients; an exploratory analysis of data from the head injury retrieval trial. Scand J Trauma Resusc Emerg Med. 2015;23:28.

13. Huber S, Cronlein M, von Matthey F, Hanschen M, Seidl F, Kirchhoff C, Biberthaler $P$, Lefering R, Huber-Wagner $\mathrm{S}$. Effect of private versus emergency medical systems transportation in trauma patients in a mostly physician based system- a retrospective multicenter study based on the TraumaRegister DGU(R). Scand J Trauma Resusc Emerg Med. 2016;24:60.

14. Bland JM, Altman DG. Statistical methods for assessing agreement between two methods of clinical measurement. Lancet (London, England). 1986;1 (8476):307-10

15. Bundesärztekammer. Richtlinie der Bundesärztekammer zur Qualitätssicherung laboratoriumsmedizinischer Untersuchungen - Rili-BÄK. Dtsch Arztebl. 2014;111(38):A-1583.

16. Florkowski C, Wallace J, Walmsley T, George P. The effect of hemolysis on current troponin assays-a confounding preanalytical variable? Clin Chem. 2010;56(7):1195-7.

17. Sodi R, Darn SM, Davison AS, Stott A, Shenkin A. Mechanism of interference by haemolysis in the cardiac troponin T immunoassay. Ann Clin Biochem. 2006;43(Pt 1):49-56.

18. Basit M, Bakshi N, Hashem M, Allebban Z, Lawson N, Rosman HS, Maciejko $J$ J. The effect of freezing and long-term storage on the stability of cardiac troponin T. Am J Clin Pathol. 2007;128(1):164-7.

19. Gillis JM, Dunselman P, Jarausch J, de Jong N, Cobbaert CM. Preanalytical storage does not affect 99th percentile cardiac troponin T concentrations measured with a high-sensitivity assay. Clin Chem. 2013;59(2):442-3.

20. Li A, Brattsand G. Stability of serum samples and hemolysis interference on the high sensitivity troponin T assay. Clin Chem Lab Med. 2011;49(2):335-6.

21. Martin J, Schuster T, Moessmer G, Kochs EF, Wagner KJ. Alterations in rotation thromboelastometry (ROTEM(R)) parameters: point-of-care testing vs analysis after pneumatic tube system transport. $\mathrm{Br} J$ Anaesth. 2012;109(4):540-5.

22. Wallin O, Soderberg J, Grankvist K, Jonsson PA, Hultdin J. Preanalytical effects of pneumatic tube transport on routine haematology, coagulation parameters, platelet function and global coagulation. Clin Chem Lab Med. 2008;46(10):1443-9.

23. Gruber MA, Felbermeir S, Lindner R, Kieninger M. Preanalytics: The (in-)stability of volatile POCT parameters and the homogeneity of blood in syringes at the market. Clin Chim Acta. 2016;457:18-23.
24. Knowles TP, Mullin RA, Hunter JA, Douce FH. Effects of syringe material, sample storage time, and temperature on blood gases and oxygen saturation in arterialized human blood samples. Respir Care. 2006;51(7):732-6.

25. Vavilala MS, Lujan SB, Qiu Q, Petroni GJ, Ballarini NM, Guadagnoli N, Depetris MA, Faguaga GA, Baggio GM, Busso LO, et al. Benchmarking Prehospital and Emergency Department Care for Argentine Children with Traumatic Brain Injury: For the South American Guideline Adherence Group. PLoS One. 2016;11(12):e0166478.

\section{Submit your next manuscript to BioMed Central and we will help you at every step:}

- We accept pre-submission inquiries

- Our selector tool helps you to find the most relevant journal

- We provide round the clock customer support

- Convenient online submission

- Thorough peer review

- Inclusion in PubMed and all major indexing services

- Maximum visibility for your research

Submit your manuscript at www.biomedcentral.com/submit
Biomed Central 